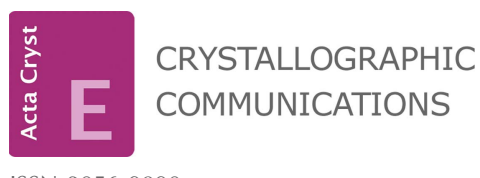

ISSN 2056-9890

Received 14 January 2020

Accepted 5 February 2020

Edited by M. Weil, Vienna University of Technology, Austria

Keywords: crystal structure; allyl; methoxyphenoxy; C- $\mathrm{H} \cdots \pi$ (ring); Hirshfeld surface.

Supporting information: this article has supporting information at journals.iucr.org/e

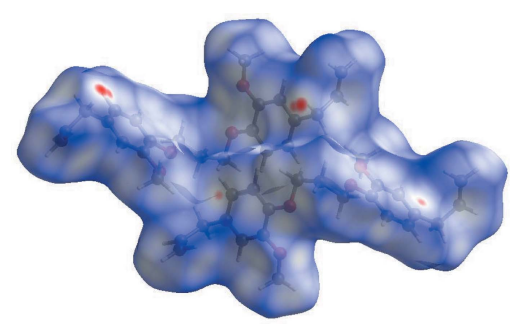

OPEN Ә ACCESS

\section{Crystal structure, Hirshfeld surface analysis and DFT studies of 1,3-bis[2-methoxy-4-(prop-2-en-1- yl)phenoxy]propane}

\author{
Abdelmaoujoud Taia, ${ }^{\mathrm{a} *}$ Mohamed Essaber, ${ }^{\mathrm{a}}$ Tuncer Hökelek, ${ }^{\mathrm{b}}$ Abdeljalil Aatif, ${ }^{\mathrm{a}}$ \\ Joel T. Mague, ${ }^{c}$ Ali Alsalme ${ }^{d}$ and Nabil Al-Zaqri ${ }^{\mathrm{e}}$
}

\begin{abstract}
a Laboratory of Molecular Chemistry, Department of Chemistry, Faculty of Sciences Semlalia, University of Cadi Ayyad, PB. 2390, 40001 Marrakech, Morocco, b Department of Physics, Hacettepe University, 06800 Beytepe, Ankara, Turkey, ${ }^{\mathbf{c}}$ Department of Chemistry, Tulane University, New Orleans, LA 70118, USA, ' Department of Chemistry, College of Science, King Saud University, P.O.Box 2455, Riyadh 11451, Saudi Arabia, and ${ }^{\mathbf{e} D e p a r t m e n t ~ o f ~ C h e m i s t r y, ~ C o l l e g e ~ o f ~}$ Science, King Saud University, PO Box 2455, Riyadh 11451, Saudi Arabia. *Correspondence e-mail: AbdelmaoujoudTaia2018@gmail.com
\end{abstract}

The asymmetric unit of the title compound, $\mathrm{C}_{23} \mathrm{H}_{28} \mathrm{O}_{4}$, comprises two halfmolecules, with the other half of each molecule being completed by the application of twofold rotation symmetry. The two completed molecules both have a V-shaped appearance but differ in their conformations. In the crystal, each independent molecule forms chains extending parallel to the $b$ axis with its symmetry-related counterparts through $\mathrm{C}-\mathrm{H} \cdots \pi($ ring) interactions. Hirshfeld surface analysis of the crystal structure indicates that the most important contributions for the crystal packing are from $\mathrm{H} \cdots \mathrm{H}(65.4 \%), \mathrm{H} \cdots \mathrm{C} / \mathrm{C} \cdots \mathrm{H}$ $(21.8 \%)$ and $\mathrm{H} \cdots \mathrm{O} / \mathrm{O} \cdots \mathrm{H}(12.3 \%)$ interactions. Optimized structures using density functional theory (DFT) at the B3LYP/6-311 G(d,p) level are compared with the experimentally determined molecular structures in the solid state. The HOMO-LUMO behaviour was elucidated to determine the energy gap.

\section{Chemical context}

Eugenol (4-allyl-2-methoxyphenol) is the main active constituent of clove oil $(75-90 \%)$ from various plants (Patra \& Saxena, 2010). The 4-allyl-2-methoxyphenol core has several active sites and provides a great responsiveness, making it an excellent precursor in the syntheses of new heterocyclic compounds (Araújo et al., 2010; Xu et al., 2006) and for the development of drugs (Sticht \& Smith, 1971). With respect to the biological applications of eugenol 4-allyl-2-methoxyphenol derivatives, it has been shown that these compounds possess potent antimicrobial (Eyambe et al., 2011), antioxidant (Nam \& Kim, 2013; Mahapatra et al., 2009; Eyambe et al., 2011), antiviral (Sun et al., 2016), anti-inflammatory (Fonsêca et al., 2016), antidiabetic and anti-leishmania (de Morais et al., 2014) properties. The suppression of melanoma growth caused by eugenol was reported by Ghosh et al. (2005), and the ability of eugenol to act as an in vivo radio-protective agent was described by Tiku et al. (2004). Derivatives of eugenol have also been reported, see, for example: Sadeghian et al. (2008); Ma et al. (2010).

As a continuation of our research devoted to the study of $o$ alkylation reactions involving eugenol derivatives, we report herein the synthesis, molecular and crystal structures of the title compound, (I). Hirshfeld surface analysis and a density functional theory (DFT) study carried out at the B3LYP/6- 
$311 \mathrm{G}(\mathrm{d}, \mathrm{p})$ level for comparison with the experimentally determined molecular structure.

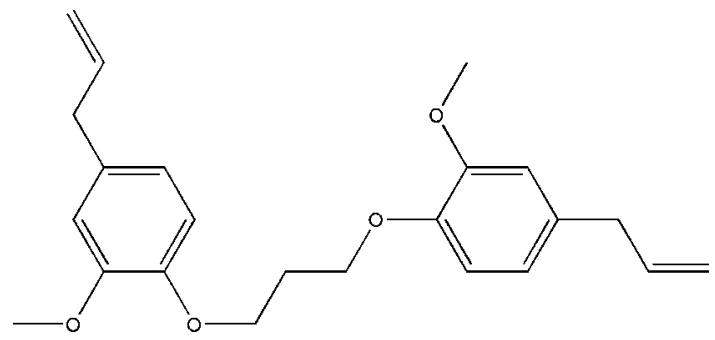

\section{Structural commentary}

The asymmetric unit of (I) comprises of two half-molecules $A$ and $B$ that are each completed by twofold rotation symmetry, with the rotation axis running through the central $\mathrm{C}$ atom $(\mathrm{C} 8$ for molecule $A$ and $\mathrm{C} 20$ for molecule $B$, respectively) of the propane bridge (Fig. 1). For steric reasons, the exocyclic substituents bound to $\mathrm{O} 1, \mathrm{O} 2, \mathrm{O} 3$ and $\mathrm{O} 4$ are approximately in trans positions, with $\mathrm{C} 1-\mathrm{C} 2-\mathrm{O} 2-\mathrm{C} 9, \mathrm{C} 2-\mathrm{C} 1-\mathrm{O} 1-\mathrm{C} 7$, $\mathrm{C} 13-\mathrm{C} 14-\mathrm{O} 4-\mathrm{C} 21$ and $\mathrm{C} 14-\mathrm{C} 13-\mathrm{O} 3-\mathrm{C} 19$ torsion angles of $-167.6(1), 175.1(1), 164.6(1)$ and $-176.7(1)^{\circ}$, respectively. The two benzene rings in each molecule are nearly perpendicular to each other, with dihedral angles of $86.74(6)^{\circ}$ for $A$ (C1-C6) and $A^{\mathrm{ii}}$, and of 88.12 (6) for $B$ (C13$\mathrm{C} 18)$ and $B^{\mathrm{i}}$, respectively (for symmetry codes, see Fig. 1 ). The two molecules have a similar V-shaped appearance but different conformations (Fig. 2).

\section{Supramolecular features}

In the crystal, chains extending parallel to the $b$ axis are formed through $\mathrm{C} 7-\mathrm{H} 7 A \cdots C g 1$ (for molecule $A$ ) and $\mathrm{C} 19-$

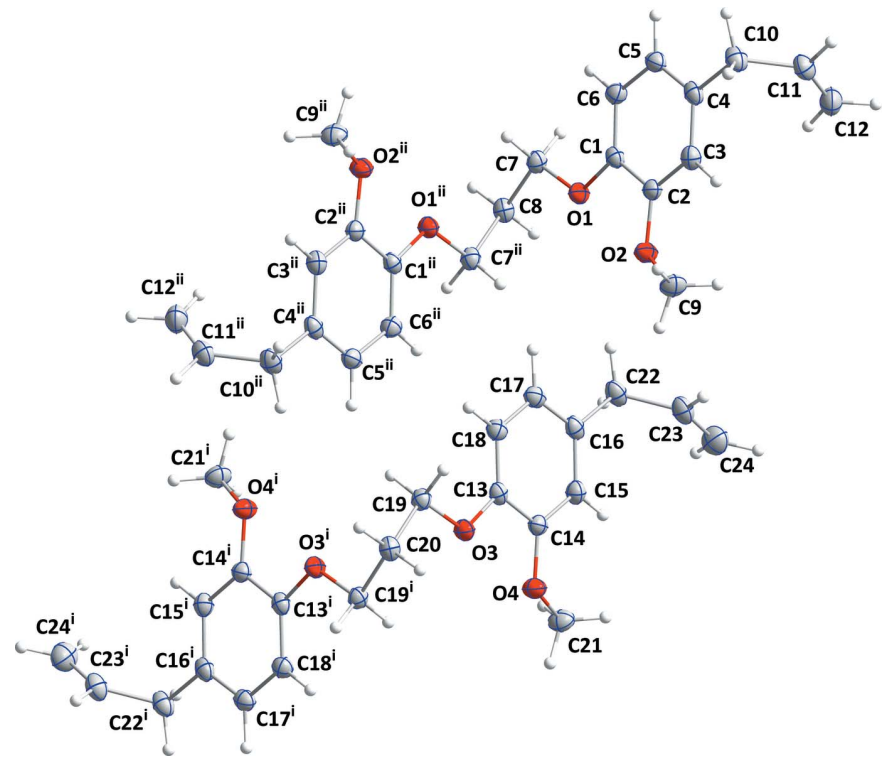

Figure 1

The two independent molecules of (I) with the atom-numbering scheme. Displacement ellipsoids are drawn at the $50 \%$ probability level. [Symmetry codes: (i) $-x+\frac{1}{2}, y,-z+\frac{1}{2}$; (ii) $-x+\frac{3}{2}, y,-z+\frac{1}{2}$.]

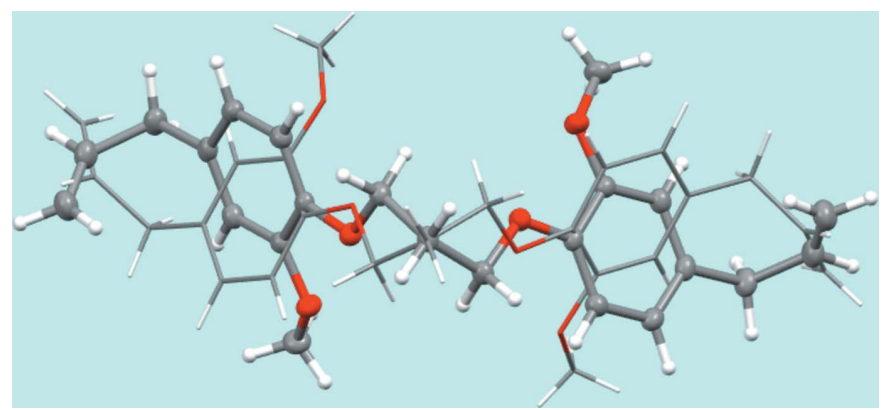

Figure 2

Overlay of the two independent half-molecules, showing their different conformations. Molecule $A$ is in light, molecule $B$ in dark colours.

H19B $\cdots C$ C 2 (for molecule B) interactions (Fig. 3, Table 1). Between the chains, only van der Waals contacts occur (Figs. 3 and 4, Table 2).

\section{Hirshfeld surface analysis}

In order to quantify the intermolecular interactions in the crystal of (I), a Hirshfeld surface (HS) analysis (Hirshfeld,

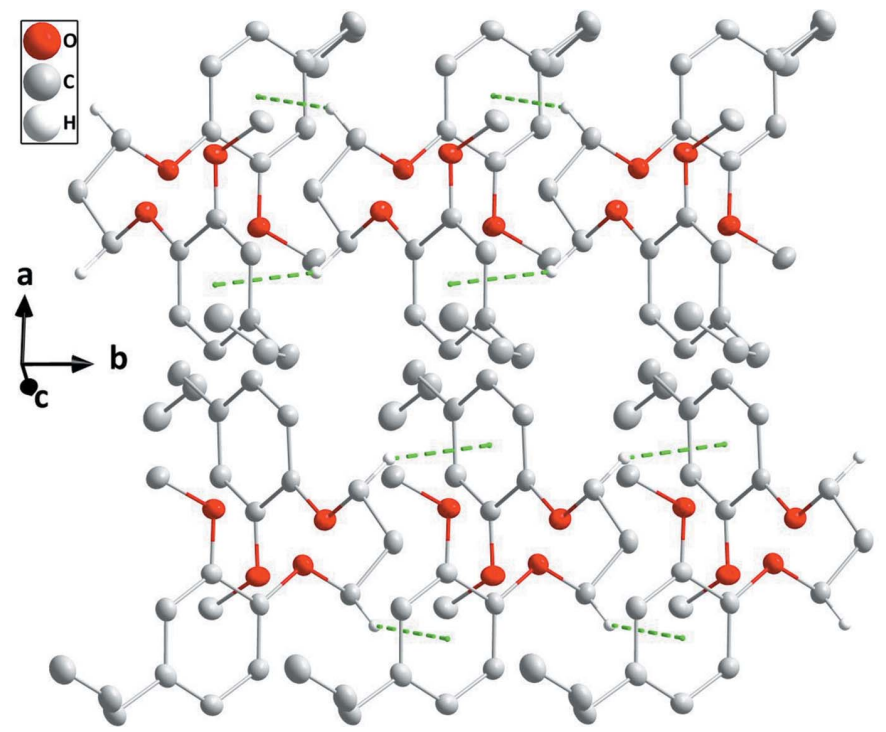

Figure 3

$\mathrm{C}-\mathrm{H} \cdots \pi$ (ring) interactions (green dashed lines) enabling the formation of molecular chains extending along the $b$-axis direction.

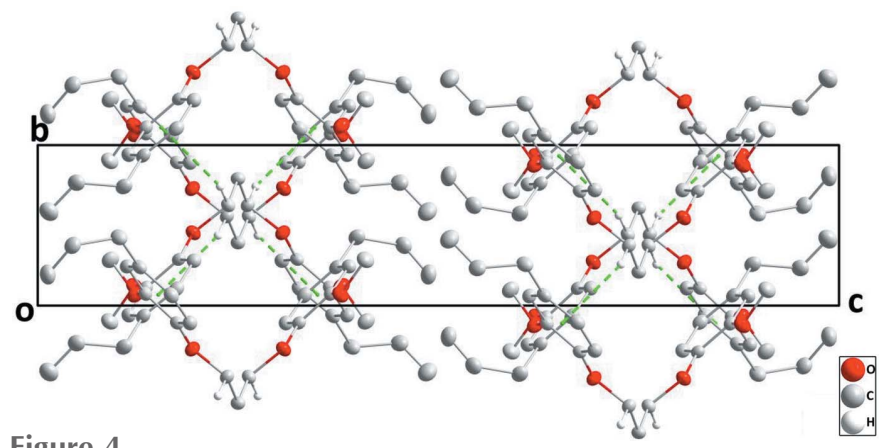

Figure 4

A partial packing diagram viewed along the $a$ axis with intermolecular interactions depicted as in Fig. 3. 
Table 1

Hydrogen-bond geometry $\left(\AA,^{\circ}\right)$.

$C g 1$ and $C g 2$ are the centroids of benzene rings $A(\mathrm{C} 1-\mathrm{C} 6)$ and $B(\mathrm{C} 13-\mathrm{C} 18)$, respectively.

\begin{tabular}{lllll}
\hline$D-\mathrm{H} \cdots A$ & $D-\mathrm{H}$ & $\mathrm{H} \cdots A$ & $D \cdots A$ & $D-\mathrm{H} \cdots A$ \\
\hline $\mathrm{C} 7-\mathrm{H} 7 A \cdots C g 1^{\mathrm{i}}$ & $1.003(16)$ & $2.759(15)$ & $3.6170(15)$ & $144.1(12)$ \\
$\mathrm{C} 19-\mathrm{H} 19 B \cdots C g 2^{\mathrm{v}}$ & $0.992(16)$ & $2.739(15)$ & $3.5816(15)$ & $143.0(11)$ \\
\hline
\end{tabular}

Symmetry codes: (i) $x, y-1, z$; (v) $x, y+1, z$.

1977; Spackman \& Jayatilaka, 2009) was carried out using Crystal Explorer 17.5 (Turner et al., 2017). In the HS plotted over $d_{\text {norm }}$ (Fig. 5), the white surface indicates contacts with distances equal to the sum of van der Waals radii, and the red and blue colours indicate distances shorter (in close contact) or longer (distinct contact) than the van der Waals radii, respectively (Venkatesan et al., 2016). The bright-red spots appearing near $\mathrm{C} 16$ and hydrogen atom $\mathrm{H} 10 B$ indicate their roles as the donor and/or acceptor groups in hydrogenbonding contacts. The shape-index of the HS is a tool to visualize possible $\pi-\pi$ stacking interactions by the appearance of adjacent red and blue triangles. The absence of such triangles suggests that there are no notable $\pi-\pi$ interactions in

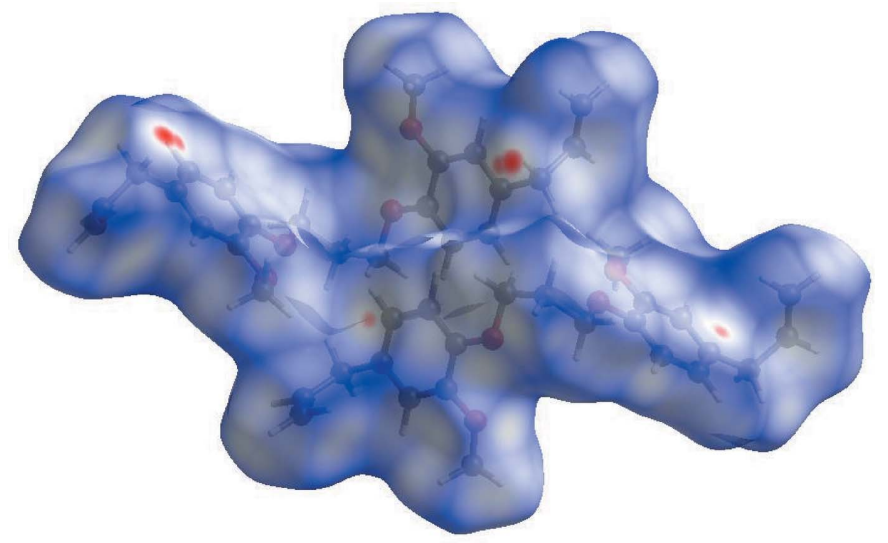

Figure 5

View of the three-dimensional Hirshfeld surface of the title compound plotted over $d_{\text {norm }}$ in the range -0.1048 to 1.1789 a.u..

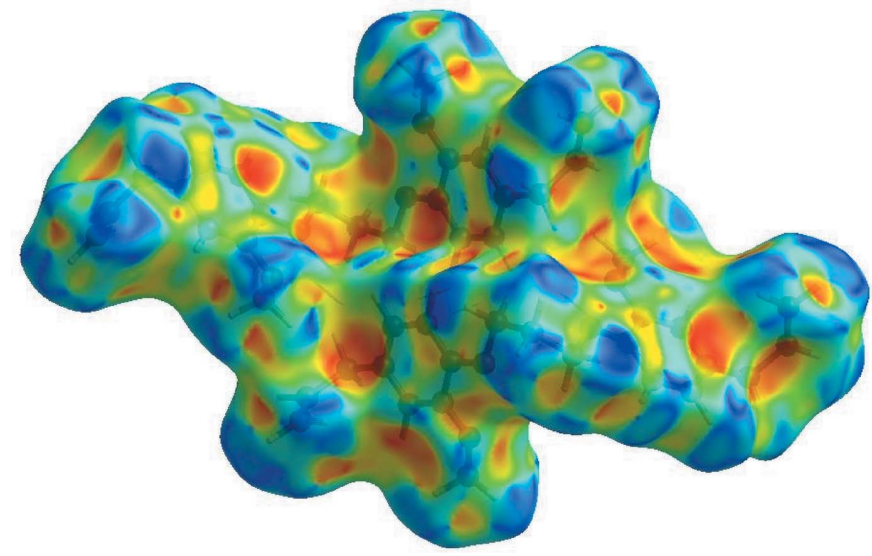

Figure 6

Hirshfeld surface of the title compound plotted over shape-index.
Table 2

Selected interatomic distances $(\AA)$.

\begin{tabular}{|c|c|c|c|}
\hline $\mathrm{O} 1 \cdots \mathrm{O} 2$ & $2.5827(13)$ & $\mathrm{C} 14 \cdots \mathrm{H} 20 B^{\mathrm{i}}$ & $2.918(15)$ \\
\hline $\mathrm{O} 2 \cdots \mathrm{O} 1$ & 2.5827 (13) & $\mathrm{C} 15 \cdots \mathrm{H} 21 A$ & $2.768(17)$ \\
\hline $\mathrm{O} 3 \cdots \mathrm{O} 4$ & 2.5885 (13) & $\mathrm{C} 15 \cdots \mathrm{H} 21 C$ & $2.784(17)$ \\
\hline $\mathrm{O} 4 \cdots \mathrm{O} 3$ & $2.5885(13)$ & $\mathrm{C} 17 \cdots \mathrm{H} 22 A^{\mathrm{v}}$ & $2.727(19)$ \\
\hline $\mathrm{O} 1 \cdots \mathrm{H} 9 C^{\mathrm{i}}$ & $2.736(18)$ & $\mathrm{C} 18 \cdots \mathrm{H} 22 A^{\mathrm{v}}$ & $2.764(18)$ \\
\hline $\mathrm{O} 1 \cdots \mathrm{H} 7 B^{\mathrm{ii}}$ & $2.618(16)$ & $\mathrm{C} 18 \cdots \mathrm{H} 19 A$ & $2.759(15)$ \\
\hline $\mathrm{O} 2 \cdots \mathrm{H} 12 A^{\mathrm{iii}}$ & $2.831(18)$ & $\mathrm{C} 18 \cdots \mathrm{H} 19 B$ & $2.739(15)$ \\
\hline $\mathrm{O} 2 \cdots \mathrm{H} 22 B$ & 2.647 (17) & C19 ․H.H18 & $2.514(16)$ \\
\hline $\mathrm{O} 2 \cdots \mathrm{H} 8 B^{\text {iv }}$ & $2.676(15)$ & $\mathrm{C} 21 \cdots \mathrm{H} 15$ & $2.501(16)$ \\
\hline $\mathrm{O} 3 \cdots \mathrm{H} 21 A^{\mathrm{v}}$ & $2.739(15)$ & 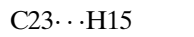 & $2.862(16)$ \\
\hline $\mathrm{O} 3 \cdots \mathrm{H} 19 A^{\mathrm{vi}}$ & $2.604(16)$ & $\mathrm{H} 3 \cdots \mathrm{H} 9 \mathrm{C}$ & $2.33(2)$ \\
\hline $\mathrm{O} 4 \cdots \mathrm{H} 24 B^{\mathrm{vii}}$ & $2.89(2)$ & $\mathrm{H} 3 \cdots \mathrm{H} 9 A$ & $2.29(2)$ \\
\hline $\mathrm{O} 4 \cdots \mathrm{H} 20 B^{\mathrm{i}}$ & $2.732(15)$ & $\mathrm{H} 5 \cdots \mathrm{H} 10 A$ & $2.37(2)$ \\
\hline $\mathrm{C} 2 \cdots \mathrm{C} 7^{\mathrm{v}}$ & $3.533(2)$ & $\mathrm{H} 6 \cdots \mathrm{H} 7 A$ & $2.24(2)$ \\
\hline $\mathrm{C} 3 \cdots \mathrm{C} 12$ & $3.282(2)$ & $\mathrm{H} 6 \cdots \mathrm{H} 7 B$ & $2.37(2)$ \\
\hline $\mathrm{C} 6 \cdots \mathrm{C} 10^{\mathrm{i}}$ & $3.582(2)$ & H6 $\cdots H 18^{\text {viii }}$ & $2.50(2)$ \\
\hline $\mathrm{C} 14 \cdots \mathrm{C} 19^{\mathrm{i}}$ & $3.555(2)$ & $\mathrm{H} 9 A \cdots \mathrm{H} 11^{\mathrm{ix}}$ & $2.40(2)$ \\
\hline $\mathrm{C} 18 \cdots \mathrm{C} 22^{\mathrm{v}}$ & $3.564(2)$ & $\mathrm{H} 9 A \cdots \mathrm{H} 12 A^{\mathrm{iii}}$ & $2.56(3)$ \\
\hline $\mathrm{C} 2 \cdots \mathrm{H} 8 B^{\mathrm{iv}}$ & $2.914(16)$ & $\mathrm{H} 9 B \cdots \mathrm{H} 22 A^{\mathrm{v}}$ & $2.52(2)$ \\
\hline $\mathrm{C} 2 \cdots \mathrm{H} 7 A^{\mathrm{v}}$ & $2.971(15)$ & $\mathrm{H} 9 B \cdots \mathrm{H} 23$ & $2.41(2)$ \\
\hline $\mathrm{C} 3 \cdots \mathrm{H} 9 \mathrm{C}$ & $2.739(18)$ & $\mathrm{H} 9 C \cdots \mathrm{H} 22 B^{\mathrm{v}}$ & $2.54(2)$ \\
\hline $\mathrm{C} 3 \cdots \mathrm{H} 12 B$ & $2.783(19)$ & $\mathrm{H} 10 A \cdots \mathrm{H} 21 A^{\mathrm{x}}$ & $2.58(2)$ \\
\hline $\mathrm{C} 3 \cdots \mathrm{H} 9 A$ & $2.806(17)$ & $\mathrm{H} 12 B \cdots \mathrm{H} 12 B^{\mathrm{iii}}$ & $2.55(3)$ \\
\hline C4. H $12 B$ & $2.728(18)$ & $\mathrm{H} 15 \cdots \mathrm{H} 21 A$ & $2.40(2)$ \\
\hline $\mathrm{C} 5 \cdots \mathrm{H} 10 B^{\mathrm{i}}$ & $2.775(19)$ & $\mathrm{H} 15 \cdots \mathrm{H} 21 C$ & $2.26(2)$ \\
\hline C6 $\cdots H 7 A$ & $2.719(15)$ & $\mathrm{H} 17 \cdots \mathrm{H} 22 B$ & $2.36(2)$ \\
\hline $\mathrm{C} 6 \cdot \cdots \mathrm{H} 7 B$ & $2.786(15)$ & $\mathrm{H} 18 \cdots \mathrm{H} 19 A$ & $2.31(2)$ \\
\hline $\mathrm{C} 6 \cdots \mathrm{H} 10 B^{\mathrm{i}}$ & 2.837 (18) & $\mathrm{H} 18 \cdots \mathrm{H} 19 B$ & $2.26(2)$ \\
\hline $\mathrm{C} 7 \cdots \mathrm{H} 6$ & $2.529(16)$ & $\mathrm{H} 21 C \cdots \mathrm{H} 23^{\mathrm{vii}}$ & $2.41(2)$ \\
\hline $\mathrm{C} 9 \cdots \mathrm{H} 3$ & $2.492(16)$ & $\mathrm{H} 22 A \cdots \mathrm{H} 24 A$ & $2.39(3)$ \\
\hline
\end{tabular}

Symmetry codes: (i) $x, y-1, z$; (ii) $-x+\frac{3}{2}, y,-z+\frac{1}{2}$; (iii) $-x+2,-y,-z+1$; (iv) $-x+\frac{3}{2}, y+1,-z+\frac{1}{2}$; (v) $x, y+1, z$; (vi) $-x+\frac{1}{2}, y,-z+\frac{1}{2}$; (vii) $-x+1,-y,-z+1$; (viii) $-x+\frac{3}{2}, y-1,-z+\frac{1}{2}$; (ix) $-x+2,-y+1,-z+1$; (x) $x+1, y+1, z$.

(I) (Fig. 6). The overall two-dimensional fingerprint plot, Fig. $7 a$, and those delineated into $\mathrm{H} \cdots \mathrm{H}, \mathrm{H} \cdots \mathrm{C} / \mathrm{C} \cdots \mathrm{H}$ and
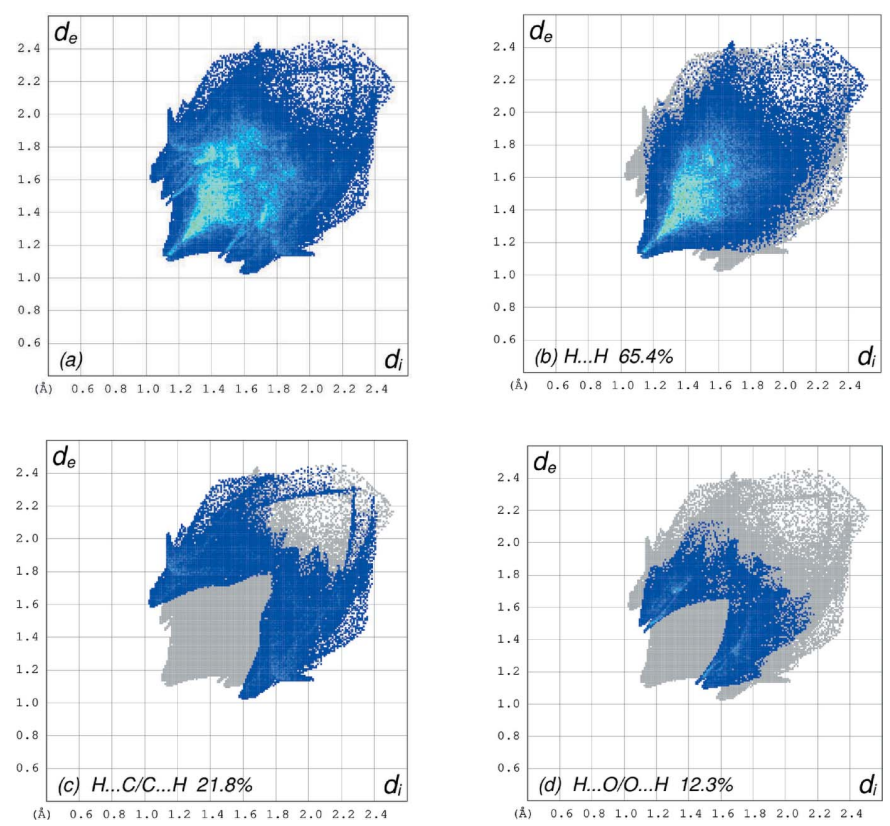

Figure 7

The full two-dimensional fingerprint plots for the title compound, showing $(a)$ all interactions, and those delineated into $(b) \mathrm{H} \cdots \mathrm{H},(c)$ $\mathrm{H} \cdots \mathrm{C} / \mathrm{C} \cdots \mathrm{H}$ and $(d) \mathrm{H} \cdots \mathrm{O} / \mathrm{O} \cdots \mathrm{H}$ interactions. The $d_{\mathrm{i}}$ and $d_{\mathrm{e}}$ values are the closest internal and external distances (in $\AA$ ) from given points on the Hirshfeld surface contacts. 
Table 3

Comparison of selected bond lengths and angles $\left(\AA,{ }^{\circ}\right)$ in the experinentally determined and computed molecular structures.

\begin{tabular}{lll}
\hline Bonds/angles & X-ray (this study) & B3LYP/6-311G(d,p) \\
\hline O1-C1 & $1.3704(15)$ & 1.38958 \\
O1-C7 & $1.4342(15)$ & 1.46082 \\
O2-C2 & $1.3679(15)$ & 1.39236 \\
O2-C9 & $1.4280(16)$ & 1.44976 \\
O3-C13 & $1.3658(15)$ & 1.39978 \\
O3-C19 & $1.4359(15)$ & 1.47837 \\
O4-C14 & $1.3697(15)$ & 1.39894 \\
O4-C21 & $1.4289(16)$ & 1.45321 \\
& & \\
C1-O1-C7 & $116.96(10)$ & 118.14221 \\
C2-O2-C9 & $116.34(10)$ & 117.63310 \\
C13-O3-C19 & $116.88(10)$ & 117.32223 \\
C14-O4-C21 & $116.03(10)$ & 117.85841 \\
O1-C1-C6 & $125.38(11)$ & 124.87388 \\
O1-C1-C2 & $115.50(11)$ & 116.13060 \\
C6-C1-C2 & $119.12(11)$ & 118.99394 \\
O2-C2-C3 & $124.79(12)$ & 124.29736 \\
O2-C2-C1 & $115.21(11)$ & 115.78966 \\
\hline
\end{tabular}

$\mathrm{H} \cdots \mathrm{O} / \mathrm{O} \cdots \mathrm{H}$ contacts (McKinnon et al., 2007) are illustrated in Fig. $7 b-d$, respectively, together with their relative contributions to the Hirshfeld surface. The most important intermolecular interactions (Table 2) are $\mathrm{H} \cdots \mathrm{H}$ contacts, contributing $65.4 \%$ to the overall crystal packing, which is reflected in Fig. $7 b$ as widely scattered points of high density due to the large hydrogen content of the molecule with the tip at $d_{\mathrm{e}}=d_{\mathrm{i}}=1.11 \AA \AA$. In the presence of $\mathrm{C}-\mathrm{H} \cdots \pi$ interactions, pairs of characteristic wings with spikes at the tips at $d_{\mathrm{e}}+d_{\mathrm{i}}=$ $2.62 \AA$ are seen in the fingerprint plot delineated into $\mathrm{H} \cdots \mathrm{C} /$ C. $\cdot \mathrm{H}$ contacts, Fig. $7 c(21.8 \%$ contribution to the HS). Finally, the thin and thick pairs of scattered wings in the fingerprint plot delineated into $\mathrm{H} \cdots \mathrm{O} / \mathrm{O} \cdots \mathrm{H}$ contacts $(12.3 \%$ contribution), Fig. $7 d$, have a symmetrical distribution of points with the edges at $d_{\mathrm{e}}+d_{\mathrm{i}}=2.55$ and $2.58 \AA$.

Hirshfeld surface representations with the function $d_{\text {norm }}$ plotted onto the surface are shown for the $\mathrm{H} \cdots \mathrm{H}, \mathrm{H} \cdots \mathrm{C} /$

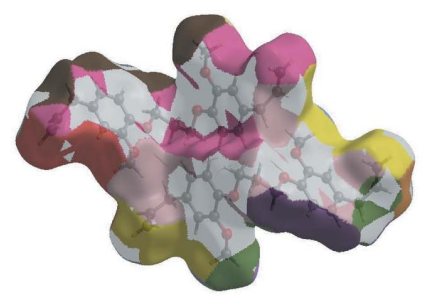

(a)

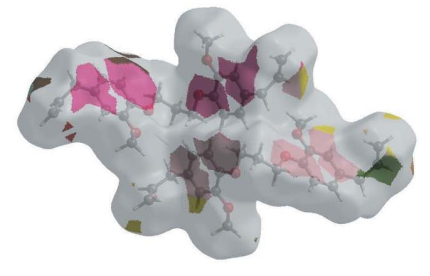

(b)

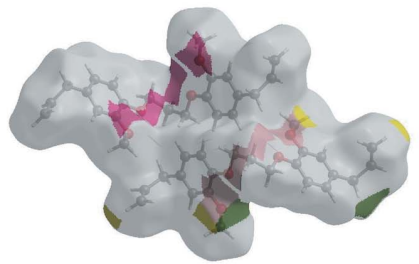

(c)

Figure 8

The Hirshfeld surface representations with the function $d_{\text {norm }}$ plotted onto the surface for $(a) \mathrm{H} \cdots \mathrm{H},(b) \mathrm{H} \cdots \mathrm{C} / \mathrm{C} \cdots \mathrm{H}$ and $(c) \mathrm{H} \cdots \mathrm{O} / \mathrm{O} \cdots \mathrm{H}$ interactions.
Table 4

Calculated energies and other calculated data for (I).

\begin{tabular}{lr}
\hline Total Energy, $T E(\mathrm{eV})$ & -32558 \\
$\mathrm{E}_{\text {HOMo }}(\mathrm{eV})$ & -5.4058 \\
$\mathrm{E}_{\mathrm{Lumo}}(\mathrm{eV})$ & -0.1807 \\
$\mathrm{Gap}, \Delta E(\mathrm{eV})$ & 5.2251 \\
Dipole moment, $\mu$ (Debye) & 2.8076 \\
Ionization potential, $I(\mathrm{eV})$ & 5.4058 \\
Electron affinity, $A$ & 0.1807 \\
Electronegativity, $\chi$ & 2.7932 \\
Hardness, $\eta$ & 2.6126 \\
Electrophilicity index, $\omega$ & 1.4932 \\
Softness, $\sigma$ & 0.3828 \\
Fraction of electrons transferred, $\Delta N$ & 0.8051 \\
\hline
\end{tabular}

$\mathrm{C} \cdots \mathrm{H}$ and $\mathrm{H} \cdots \mathrm{O} / \mathrm{O} \cdots \mathrm{H}$ interactions in Fig. $8 a-c$, respectively.

The large number of $\mathrm{H} \cdots \mathrm{H}, \mathrm{H} \cdots \mathrm{C} / \mathrm{C} \cdots \mathrm{H}$ and $\mathrm{H} \cdots \mathrm{O} /$ $\mathrm{O} \cdots \mathrm{H}$ intermolecular contacts suggest that these weak interactions play major roles in the crystal packing (Hathwar et al., 2015).

\section{DFT calculations}

The density functional theory (DFT) optimized molecular structures of (I) were computed in the gas phase on the basis of standard B3LYP functionals and 6-311 G(d,p) basis-set calculations (Becke, 1993) as implemented in GAUSSIAN 09 (Frisch et al., 2009). The theoretical and experimental results for molecule $A$ are in good agreement (Table 3).
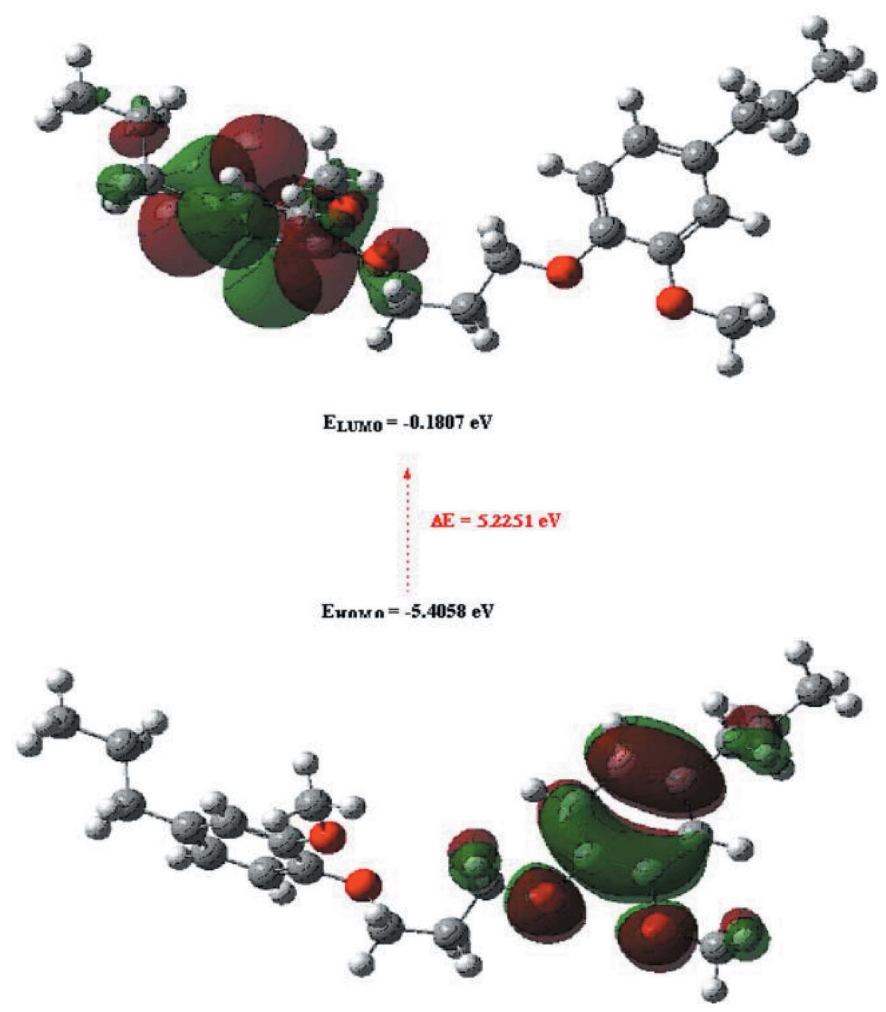

Figure 9

The shapes of HOMO and LUMO orbitals in one of the molecules in (I). 
Table 5

Experimental details.

\begin{tabular}{|c|c|}
\hline \multicolumn{2}{|l|}{ Crystal data } \\
\hline Chemical formula & $\mathrm{C}_{23} \mathrm{H}_{28} \mathrm{O}_{4}$ \\
\hline$M_{\mathrm{r}}$ & 368.45 \\
\hline Crystal system, space group & Monoclinic, $P 2 / n$ \\
\hline Temperature (K) & 150 \\
\hline$a, b, c(\AA)$ & $15.4741(5), 5.0224(2), 25.5180(9)$ \\
\hline$\beta\left({ }^{\circ}\right)$ & $99.858(2)$ \\
\hline$V\left(\AA^{6}\right)$ & $1953.90(12)$ \\
\hline$Z$ & 4 \\
\hline Radiation type & $\mathrm{Cu} \mathrm{K \alpha}$ \\
\hline$\mu\left(\mathrm{mm}^{-1}\right)$ & 0.68 \\
\hline Crystal size (mm) & $0.37 \times 0.27 \times 0.08$ \\
\hline \multicolumn{2}{|l|}{ Data collection } \\
\hline Diffractometer & $\begin{array}{l}\text { Bruker D8 VENTURE PHOTON } \\
100 \text { CMOS }\end{array}$ \\
\hline Absorption correction & $\begin{array}{l}\text { Multi-scan (SADABS; Krause et } \\
\quad \text { al., 2015) }\end{array}$ \\
\hline$T_{\min }, T_{\max }$ & $0.79,0.95$ \\
\hline $\begin{array}{l}\text { No. of measured, independent and } \\
\text { observed }[I>2 \sigma(I)] \text { reflections }\end{array}$ & $13935,3756,3049$ \\
\hline$R_{\text {int }}$ & 0.033 \\
\hline$(\sin \theta / \lambda)_{\max }\left(\AA^{-1}\right)$ & 0.618 \\
\hline \multicolumn{2}{|l|}{ Refinement } \\
\hline$R\left[F^{2}>2 \sigma\left(F^{2}\right)\right], w R\left(F^{2}\right), S$ & $0.041,0.109,1.05$ \\
\hline No. of reflections & 3756 \\
\hline No. of parameters & 358 \\
\hline $\mathrm{H}$-atom treatment & All H-atom parameters refined \\
\hline$\Delta \rho_{\max }, \Delta \rho_{\min }\left(\mathrm{e} \AA^{-3}\right)$ & $0.19,-0.23$ \\
\hline
\end{tabular}

Computer programs: APEX3 and SAINT (Bruker, 2016), SHELXT (Sheldrick, 2015a), SHELXL2018 (Sheldrick, 2015b), DIAMOND (Brandenburg \& Putz, 2012) and publCIF (Westrip, 2010).

If the energy gap $\Delta E$ between the highest occupied molecular orbital (HOMO) and the lowest unoccupied molecular orbital (LUMO) is small, the molecule is highly polarizable and has high chemical reactivity. Numerical values of $E_{\text {НОмО }}$ and $E_{\mathrm{LUMO}}, \Delta E=E_{\mathrm{LUMO}}-E_{\mathrm{HOMO}}$, electronegativity $(\chi)$, hardness $(\eta)$, potential $(\mu)$, electrophilicity $(\omega)$ and softness $(\sigma)$ for (I) are collated in Table 4. The significance of $\eta$ and $\sigma$ is to evaluate both the reactivity and stability. The shapes of the HOMO and the LUMO of molecule $A$, together with their energy levels are shown in Fig. 9.

\section{Synthesis and crystallization}

1,3-Dibromopropane $(0.2 \mathrm{ml}, 1.61 \mathrm{mmol})$ was added to a solution of eugenol $(0.5 \mathrm{ml}, 3.23 \mathrm{mmol})$, tetrabutylammonium chloride $(50 \mathrm{mg})$ and sodium hydroxide solution $(5 \%)$ in benzene as solvent $(20 \mathrm{ml})$. The mixture was stirred at $293 \mathrm{~K}$ for $6 \mathrm{~h}$, and then was extracted three times with dichloromethane $(15 \mathrm{ml})$. The residue was purified by column chromatography on silica gel using a mixture of hexane/ethyl acetate $(v / v=97 / 3)$ as eluent. Colourless crystals were isolated when the solvent was allowed to evaporate (yield: $86 \%$ ).

\section{Refinement}

Details including crystal data, data collection and refinement are summarized in Table 5. Hydrogen atoms were located in a difference-Fourier map and were refined freely.

\section{Funding information}

The support of NSF-MRI grant No. 1228232 for the purchase of the diffractometer and Tulane University for support of the Tulane Crystallography Laboratory are gratefully acknowledged. TH is grateful to Hacettepe University Scientific Research Project Unit (grant No. 013 D04 602 004). The Researchers Supporting Project (No. RSP-2019/78) King Saudi University, Riyadh, Saudi Arabia also supported this work.

\section{References}

Araújo, J. D. P., Grande, C. A. \& Rodrigues, A. E. (2010). Chem. Eng. Res. Des. 88, 1024-1032.

Becke, A. D. (1993). J. Chem. Phys. 98, 5648-5652.

Brandenburg, K. \& Putz, H. (2012). DIAMOND, Crystal Impact GbR, Bonn, Germany.

Bruker (2016). APEX3 and SAINT. Bruker AXS, Inc., Madison, Wisconsin, USA.

Eyambe, G., Canales, L. \& Banik, B. K. (2011). Heterocyclic Lett. 1, 154-157.

Fonsêca, D. V., Salgado, P. R. R., Neto, H. C. A., Golzio, A. M. F. O., Caldas, M. R. D. F., Melo, C. G. F., Leite, F. C., Piuvezam, M. R., Pordeus, L. C. D., Barbosa, J. M. F. \& Almeida, R. N. (2016). Int. Immunopharmacol. 38, 402-408.

Frisch, M. J., Trucks, G. W., Schlegel, H. B., Scuseria, G. E., Robb, M. A., Cheeseman, J. R., et al. (2009). GAUSSIAN09. Gaussian Inc., Wallingford, CT, USA.

Ghosh, R., Nadiminty, N., Fitzpatrick, J. E., Alworth, W. L., Slaga, T. J. \& Kumar, A. P. (2005). J. Biol. Chem. 280, 5812-5819.

Hathwar, V. R., Sist, M., Jørgensen, M. R. V., Mamakhel, A. H., Wang, X., Hoffmann, C. M., Sugimoto, K., Overgaard, J. \& Iversen, B. B. (2015). IUCrJ, 2, 563-574.

Hirshfeld, H. L. (1977). Theor. Chim. Acta, 44, 129-138.

Krause, L., Herbst-Irmer, R., Sheldrick, G. M. \& Stalke, D. (2015). J. Appl. Cryst. 48, 3-10.

Ma, Y.-T., Li, H.-Q., Shi, X.-W., Zhang, A.-L. \& Gao, J.-M. (2010). Acta Cryst. E66, o2946.

Mahapatra, S. K., Chakraborty, S. P., Majumdar, S., Bag, B. G. \& Roy, S. (2009). Eur. J. Pharmacol. 623, 132-140.

McKinnon, J. J., Jayatilaka, D. \& Spackman, M. A. (2007). Chem. Commun. pp. 3814-3816.

Morais, S. M. de, Vila-Nova, N. S., Bevilaqua, C. M. L., Rondon, F. C., Lobo, C. H., Moura, A. de A. A. N., Sales, A. D., Rodrigues, A. P. R., de Figuereido, J. R., Campello, C. C., Wilson, M. E. \& de Andrade, H. F. (2014). Bioorg. Med. Chem. 22, 6250-6255.

Nam, H. \& Kim, M.-M. (2013). Food \& Chem. Toxicol. 55, 106-112.

Patra, A. K. \& Saxena, J. (2010). Phytochemistry, 71, 1198-1222.

Sadeghian, H., Seyedi, S. M., Saberi, M. R., Arghiani, Z. \& Riazi, M. (2008). Bioorg. Med. Chem. 16, 890-901.

Sheldrick, G. M. (2015a). Acta Cryst. A71, 3-8.

Sheldrick, G. M. (2015b). Acta Cryst. C71, 3-8.

Spackman, M. A. \& Jayatilaka, D. (2009). CrystEngComm, 11, 19-32.

Sticht, F. D. \& Smith, R. M. (1971). J. Dent. Res. 50, 1531-1535.

Sun, W. J., Lv, W. J., Li, L. N., Yin, G., Hang, X. F., Xue, Y. F., Chen, J. \& Shi, Z. Q. (2016). New Biotechnol. 33, 345-354.

Tiku, A. B., Abraham, S. K. \& Kale, R. K. (2004). J. Radiat. Res. 45, 435-440.

Turner, M. J., McKinnon, J. J., Wolff, S. K., Grimwood, D. J., Spackman, P. R., Jayatilaka, D. \& Spackman, M. A. (2017). CrystalExplorer17. The University of Western Australia.

Venkatesan, P., Thamotharan, S., Ilangovan, A., Liang, H. \& Sundius, T. (2016). Spectrochim. Acta Part A, 153, 625-636.

Westrip, S. P. (2010). J. Appl. Cryst. 43, 920-925.

Xu, H. X., Delling, M., Jun, J. C. \& Clapham, D. E. (2006). Nat. Neurosci. 9, 628-635. 


\section{supporting information}

Acta Cryst. (2020). E76, 344-348 [https://doi.org/10.1107/S2056989020001681]

Crystal structure, Hirshfeld surface analysis and DFT studies of 1,3-bis[2-methoxy-4-(prop-2-en-1-yl)phenoxy]propane

Abdelmaoujoud Taia, Mohamed Essaber, Tuncer Hökelek, Abdeljalil Aatif, Joel T. Mague, Ali

Alsalme and Nabil Al-Zaqri

Computing details

Data collection: APEX3 (Bruker, 2016); cell refinement: SAINT (Bruker, 2016); data reduction: SAINT (Bruker, 2016); program(s) used to solve structure: SHELXT (Sheldrick, 2015a); program(s) used to refine structure: SHELXL2018 (Sheldrick, 2015b); molecular graphics: DIAMOND (Brandenburg \& Putz, 2012); software used to prepare material for publication: publCIF (Westrip, 2010).

1,3-Bis[2-methoxy-4-(prop-2-en-1-yl)phenoxy]propane

Crystal data

$\mathrm{C}_{23} \mathrm{H}_{28} \mathrm{O}_{4}$

$M_{r}=368.45$

Monoclinic, $P 2 / n$

$a=15.4741(5) \AA$

$b=5.0224(2) \AA$

$c=25.5180(9) \AA$

$\beta=99.858(2)^{\circ}$

$V=1953.90(12) \AA^{3}$

$Z=4$

\section{Data collection}

Bruker D8 VENTURE PHOTON 100 CMOS diffractometer

Radiation source: INCOATEC $\mathrm{I} \mu \mathrm{S}$ micro-focus source

Mirror monochromator

Detector resolution: 10.4167 pixels $\mathrm{mm}^{-1}$

$\omega$ scans

Absorption correction: multi-scan

(SADABS; Krause et al., 2015)

\section{Refinement}

Refinement on $F^{2}$

Least-squares matrix: full

$R\left[F^{2}>2 \sigma\left(F^{2}\right)\right]=0.041$

$w R\left(F^{2}\right)=0.109$

$S=1.05$

3756 reflections

358 parameters

0 restraints
$F(000)=792$

$D_{\mathrm{x}}=1.253 \mathrm{Mg} \mathrm{m}^{-3}$

$\mathrm{Cu} K \alpha$ radiation, $\lambda=1.54178 \AA$

Cell parameters from 9361 reflections

$\theta=3.5-72.4^{\circ}$

$\mu=0.68 \mathrm{~mm}^{-1}$

$T=150 \mathrm{~K}$

Plate, colourless

$0.37 \times 0.27 \times 0.08 \mathrm{~mm}$

$T_{\min }=0.79, T_{\max }=0.95$

13935 measured reflections

3756 independent reflections

3049 reflections with $I>2 \sigma(I)$

$R_{\text {int }}=0.033$

$\theta_{\text {max }}=72.4^{\circ}, \theta_{\text {min }}=3.1^{\circ}$

$h=-19 \rightarrow 18$

$k=-5 \rightarrow 6$

$l=-31 \rightarrow 29$

Primary atom site location: dual

Secondary atom site location: difference Fourier map

Hydrogen site location: difference Fourier map

All $\mathrm{H}$-atom parameters refined

$w=1 /\left[\sigma^{2}\left(F_{\mathrm{o}}^{2}\right)+(0.0537 P)^{2}+0.5337 P\right]$

where $P=\left(F_{\mathrm{o}}^{2}+2 F_{\mathrm{c}}{ }^{2}\right) / 3$

$(\Delta / \sigma)_{\max }=0.001$ 
$\Delta \rho_{\max }=0.19$ e $\AA^{-3}$

$\Delta \rho_{\min }=-0.23 \mathrm{e} \AA^{-3}$
Extinction correction: SHELXL2018 (Sheldrick, $2015 b), \mathrm{Fc}^{*}=\mathrm{kFc}\left[1+0.001 \mathrm{xFc}^{2} \lambda^{3} / \sin (2 \theta)\right]^{-1 / 4}$

Extinction coefficient: 0.0071 (4)

\section{Special details}

Geometry. All esds (except the esd in the dihedral angle between two 1.s. planes) are estimated using the full covariance matrix. The cell esds are taken into account individually in the estimation of esds in distances, angles and torsion angles; correlations between esds in cell parameters are only used when they are defined by crystal symmetry. An approximate (isotropic) treatment of cell esds is used for estimating esds involving l.s. planes.

Refinement. Refinement of $\mathrm{F}^{2}$ against ALL reflections. The weighted R-factor $\mathrm{wR}$ and goodness of fit $\mathrm{S}$ are based on $\mathrm{F}^{2}$, conventional R-factors $R$ are based on $F$, with $F$ set to zero for negative $\mathrm{F}^{2}$. The threshold expression of $\mathrm{F}^{2}>2 \operatorname{sigma}\left(\mathrm{F}^{2}\right)$ is used only for calculating R-factors(gt) etc. and is not relevant to the choice of reflections for refinement. R-factors based on $\mathrm{F}^{2}$ are statistically about twice as large as those based on F, and R- factors based on ALL data will be even larger.

Fractional atomic coordinates and isotropic or equivalent isotropic displacement parameters $\left(\hat{A}^{2}\right)$

\begin{tabular}{|c|c|c|c|c|}
\hline & $x$ & $y$ & $z$ & $U_{\text {iso }} * / U_{\text {eq }}$ \\
\hline $\mathrm{O} 1$ & $0.82230(6)$ & $-0.27068(18)$ & $0.30688(3)$ & $0.0222(2)$ \\
\hline $\mathrm{O} 2$ & $0.79368(6)$ & $0.06905(18)$ & $0.37794(4)$ & $0.0249(2)$ \\
\hline $\mathrm{O} 3$ & $0.32386(6)$ & $0.45366(18)$ & $0.30591(3)$ & $0.0223(2)$ \\
\hline $\mathrm{O} 4$ & $0.29863(6)$ & 0.12078 (19) & $0.37925(4)$ & $0.0258(2)$ \\
\hline $\mathrm{C} 1$ & $0.89151(8)$ & $-0.1081(2)$ & $0.32631(5)$ & 0.0194 \\
\hline $\mathrm{C} 2$ & $0.87636(8)$ & $0.0744(2)$ & $0.36573(5)$ & 0.0203 \\
\hline $\mathrm{C} 3$ & $0.94260(9)$ & $0.2442(3)$ & $0.38859(5)$ & $0.0229(3)$ \\
\hline $\mathrm{H} 3$ & $0.9308(10)$ & $0.372(3)$ & $0.4158(6)$ & $0.026(4)^{*}$ \\
\hline $\mathrm{C} 4$ & $1.02517(8)$ & $0.2399(3)$ & $0.37294(5)$ & $0.0227(3)$ \\
\hline $\mathrm{C} 5$ & $1.03861(8)$ & $0.0650(3)$ & $0.33324(5)$ & 0.0234 \\
\hline H5 & $1.0965(11)$ & $0.061(3)$ & $0.3204(6)$ & $0.028(4)^{*}$ \\
\hline C6 & $0.97202(8)$ & $-0.1084(3)$ & $0.30986(5)$ & $0.0226(3)$ \\
\hline H6 & $0.9834(10)$ & $-0.232(3)$ & $0.2825(6)$ & $0.028(4)^{*}$ \\
\hline $\mathrm{C} 7$ & $0.83297(8)$ & $-0.4437(3)$ & $0.26374(5)$ & $0.0219(3)$ \\
\hline $\mathrm{H} 7 \mathrm{~A}$ & $0.8849(10)$ & $-0.562(3)$ & $0.2749(6)$ & $0.023(4)^{*}$ \\
\hline $\mathrm{H} 7 \mathrm{~B}$ & $0.8441(10)$ & $-0.334(3)$ & $0.2325(6)$ & $0.025(4)^{*}$ \\
\hline $\mathrm{C} 8$ & 0.750000 & $-0.6078(4)$ & 0.250000 & $0.0224(4)$ \\
\hline $\mathrm{H} 8 \mathrm{~B}$ & $0.7581(11)$ & $-0.722(3)$ & $0.2188(6)$ & $0.033(4)^{*}$ \\
\hline C9 & $0.77076(10)$ & $0.2853(3)$ & $0.40922(6)$ & $0.0289(3)$ \\
\hline H9A & $0.8027(11)$ & $0.277(3)$ & $0.4452(7)$ & $0.034(4)^{*}$ \\
\hline H9B & $0.7090(11)$ & $0.260(3)$ & $0.4097(6)$ & $0.028(4)^{*}$ \\
\hline $\mathrm{H} 9 \mathrm{C}$ & $0.7809(11)$ & $0.458(4)$ & $0.3922(7)$ & $0.036(4)^{*}$ \\
\hline $\mathrm{C} 10$ & $1.09695(9)$ & $0.4256(3)$ & $0.39847(6)$ & 0.0280 \\
\hline $\mathrm{H} 10 \mathrm{~A}$ & $1.1474(11)$ & $0.420(3)$ & $0.3779(6)$ & $0.035(4)^{*}$ \\
\hline H10B & $1.0731(12)$ & $0.613(4)$ & $0.3959(7)$ & $0.040(5)^{*}$ \\
\hline C11 & $1.13272(9)$ & $0.3754(3)$ & $0.45635(6)$ & 0.0298 \\
\hline H11 & $1.1823(13)$ & $0.494(4)$ & $0.4714(7)$ & $0.052(5)^{*}$ \\
\hline $\mathrm{C} 12$ & $1.10801(11)$ & $0.1937(3)$ & $0.48750(6)$ & 0.0355 \\
\hline $\mathrm{H} 12 \mathrm{~A}$ & $1.1352(12)$ & $0.181(4)$ & $0.5248(7)$ & $0.044(5)^{*}$ \\
\hline H12B & $1.0621(13)$ & $0.067(4)$ & $0.4757(7)$ & $0.047(5)^{*}$ \\
\hline $\mathrm{C} 13$ & $0.39264(8)$ & $0.2882(2)$ & $0.32379(5)$ & 0.0198 \\
\hline $\mathrm{C} 14$ & $0.37979(8)$ & $0.1110(2)$ & $0.36473(5)$ & $0.0204(3)$ \\
\hline
\end{tabular}




$\begin{array}{lllll}\text { C15 } & 0.44651(9) & -0.0598(3) & 0.38657(5) & 0.0230(3) \\ \text { H15 } & 0.4371(11) & -0.180(3) & 0.4150(6) & 0.032(4)^{*} \\ \text { C16 } & 0.52658(8) & -0.0646(3) & 0.36808(5) & 0.0229(3) \\ \text { C17 } & 0.53771(8) & 0.1037(3) & 0.32686(5) & 0.0235(3) \\ \text { H17 } & 0.5958(10) & 0.104(3) & 0.3123(6) & 0.026(4)^{*} \\ \text { C18 } & 0.47108(8) & 0.2800(3) & 0.30487(5) & 0.0224(3) \\ \text { H18 } & 0.4801(10) & 0.400(3) & 0.2759(6) & 0.023(4)^{*} \\ \text { C19 } & 0.33292(8) & 0.6261(3) & 0.26230(5) & 0.0213(3) \\ \text { H19A } & 0.3426(10) & 0.514(3) & 0.2309(6) & 0.023(4)^{*} \\ \text { H19B } & 0.3849(10) & 0.743(3) & 0.2722(6) & 0.026(4)^{*} \\ \text { C20 } & 0.250000 & 0.7913(4) & 0.250000 & 0.0223(4) \\ \text { H20B } & 0.2441(11) & 0.908(3) & 0.2806(6) & 0.031(4)^{*} \\ \text { C21 } & 0.27648(10) & -0.0984(3) & 0.41011(6) & 0.0289(3) \\ \text { H21A } & 0.2830(11) & -0.273(3) & 0.3913(6) & 0.034(4)^{*} \\ \text { H21B } & 0.2159(12) & -0.073(3) & 0.4127(6) & 0.036(4)^{*} \\ \text { H21C } & 0.3109(11) & -0.096(3) & 0.4452(7) & 0.033(4)^{*} \\ \text { C22 } & 0.59762(9) & -0.2578(3) & 0.39229(6) & 0.0275(3) \\ \text { H22A } & 0.5774(11) & -0.442(4) & 0.3845(7) & 0.037(4)^{*} \\ \text { H22B } & 0.6510(11) & -0.225(3) & 0.3753(6) & 0.034(4)^{*} \\ \text { C23 } & 0.62171(9) & -0.2341(3) & 0.45158(6) & 0.0300(3) \\ \text { H23 } & 0.6524(13) & -0.064(4) & 0.4645(7) & 0.050(5)^{*} \\ \text { C24 } & 0.60596(11) & -0.4141(3) & 0.48616(7) & 0.0386(4) \\ \text { H24A } & 0.5776(13) & -0.581(4) & 0.4740(8) & 0.051(5)^{*} \\ \text { H24B } & 0.6230(13) & -0.389(4) & 0.5240(8) & 0.050(5)^{*} \\ & & & & \end{array}$

Atomic displacement parameters $\left(\AA^{2}\right)$

\begin{tabular}{lllllll}
\hline & $U^{11}$ & $U^{22}$ & $U^{33}$ & $U^{12}$ & $U^{13}$ & $U^{23}$ \\
\hline O1 & $0.0208(4)$ & $0.0227(5)$ & $0.0230(5)$ & $-0.0028(4)$ & $0.0034(3)$ & $-0.0050(4)$ \\
O2 & $0.0209(5)$ & $0.0269(5)$ & $0.0276(5)$ & $0.0002(4)$ & $0.0063(4)$ & $-0.0056(4)$ \\
O3 & $0.0212(4)$ & $0.0223(5)$ & $0.0232(5)$ & $0.0035(4)$ & $0.0032(3)$ & $0.0049(4)$ \\
O4 & $0.0225(5)$ & $0.0274(5)$ & $0.0289(5)$ & $0.0031(4)$ & $0.0084(4)$ & $0.0061(4)$ \\
C1 & $0.0191(6)$ & $0.0183(6)$ & $0.0197(6)$ & $-0.0008(5)$ & $0.0002(5)$ & $0.0022(5)$ \\
C2 & $0.0200(6)$ & $0.0215(6)$ & $0.0193(6)$ & $0.0012(5)$ & $0.0031(5)$ & $0.0022(5)$ \\
C3 & $0.0256(7)$ & $0.0208(6)$ & $0.0213(6)$ & $0.0009(5)$ & $0.0010(5)$ & $-0.0006(5)$ \\
C4 & $0.0221(6)$ & $0.0215(6)$ & $0.0224(6)$ & $-0.0019(5)$ & $-0.0015(5)$ & $0.0046(5)$ \\
C5 & $0.0196(6)$ & $0.0265(7)$ & $0.0240(6)$ & $-0.0004(5)$ & $0.0033(5)$ & $0.0045(6)$ \\
C6 & $0.0238(6)$ & $0.0224(6)$ & $0.0216(6)$ & $0.0016(5)$ & $0.0041(5)$ & $0.0002(5)$ \\
C7 & $0.0238(7)$ & $0.0203(6)$ & $0.0209(6)$ & $0.0012(5)$ & $0.0023(5)$ & $-0.0026(5)$ \\
C8 & $0.0247(9)$ & $0.0188(8)$ & $0.0223(9)$ & 0.000 & $0.0001(7)$ & 0.000 \\
C9 & $0.0293(7)$ & $0.0278(7)$ & $0.0314(8)$ & $0.0045(6)$ & $0.0100(6)$ & $-0.0045(6)$ \\
C10 & $0.0265(7)$ & $0.0263(7)$ & $0.0293(7)$ & $-0.0065(6)$ & $-0.0006(6)$ & $0.0028(6)$ \\
C11 & $0.0276(7)$ & $0.0308(7)$ & $0.0285(7)$ & $-0.0026(6)$ & $-0.0015(5)$ & $-0.0040(6)$ \\
C12 & $0.0367(8)$ & $0.0419(9)$ & $0.0258(8)$ & $-0.0008(7)$ & $-0.0007(6)$ & $0.0021(7)$ \\
C13 & $0.0207(6)$ & $0.0174(6)$ & $0.0199(6)$ & $0.0013(5)$ & $0.0000(5)$ & $-0.0022(5)$ \\
C14 & $0.0197(6)$ & $0.0210(6)$ & $0.0203(6)$ & $-0.0007(5)$ & $0.0033(5)$ & $-0.0027(5)$ \\
C15 & $0.0248(6)$ & $0.0221(6)$ & $0.0212(6)$ & $0.0019(5)$ & $0.0013(5)$ & $0.0013(5)$ \\
C16 & $0.0227(6)$ & $0.0219(6)$ & $0.0222(6)$ & $0.0013(5)$ & $-0.0015(5)$ & $-0.0052(5)$
\end{tabular}




\begin{tabular}{lllllll}
\hline & & & & & \\
C17 & $0.0209(6)$ & $0.0240(6)$ & $0.0254(7)$ & $-0.0008(5)$ & $0.0032(5)$ & $-0.0048(5)$ \\
C18 & $0.0227(6)$ & $0.0217(6)$ & $0.0225(6)$ & $-0.0020(5)$ & $0.0033(5)$ & $-0.0004(5)$ \\
C19 & $0.0226(6)$ & $0.0204(6)$ & $0.0206(6)$ & $-0.0004(5)$ & $0.0026(5)$ & $0.0027(5)$ \\
C20 & $0.0249(9)$ & $0.0181(8)$ & $0.0224(9)$ & 0.000 & $0.0001(7)$ & 0.000 \\
C21 & $0.0297(7)$ & $0.0280(7)$ & $0.0308(8)$ & $-0.0009(6)$ & $0.0104(6)$ & $0.0053(6)$ \\
C22 & $0.0253(7)$ & $0.0252(7)$ & $0.0295(7)$ & $0.0055(6)$ & $-0.0019(5)$ & $-0.0036(6)$ \\
C23 & $0.0282(7)$ & $0.0280(7)$ & $0.0311(7)$ & $0.0040(6)$ & $-0.0029(6)$ & $0.0007(6)$ \\
C24 & $0.0411(9)$ & $0.0364(9)$ & $0.0381(9)$ & $0.0078(7)$ & $0.0059(7)$ & $0.0063(7)$ \\
\hline
\end{tabular}

Geometric parameters $\left(\AA,{ }^{\circ}\right)$

\begin{tabular}{|c|c|c|c|}
\hline $\mathrm{O} 1-\mathrm{C} 1$ & $1.3704(15)$ & $\mathrm{C} 11-\mathrm{C} 12$ & $1.310(2)$ \\
\hline $\mathrm{O} 1-\mathrm{C} 7$ & $1.4342(15)$ & $\mathrm{C} 11-\mathrm{H} 11$ & $0.99(2)$ \\
\hline $\mathrm{O} 2-\mathrm{C} 2$ & $1.3679(15)$ & $\mathrm{C} 12-\mathrm{H} 12 \mathrm{~A}$ & 0.975 (19) \\
\hline $\mathrm{O} 2-\mathrm{C} 9$ & $1.4280(16)$ & $\mathrm{C} 12-\mathrm{H} 12 \mathrm{~B}$ & $0.96(2)$ \\
\hline $\mathrm{O} 3-\mathrm{C} 13$ & $1.3658(15)$ & $\mathrm{C} 13-\mathrm{C} 18$ & $1.3816(18)$ \\
\hline $\mathrm{O} 3-\mathrm{C} 19$ & $1.4359(15)$ & $\mathrm{C} 13-\mathrm{C} 14$ & $1.4121(17)$ \\
\hline $\mathrm{O} 4-\mathrm{C} 14$ & $1.3697(15)$ & $\mathrm{C} 14-\mathrm{C} 15$ & $1.3840(18)$ \\
\hline $\mathrm{O} 4-\mathrm{C} 21$ & $1.4289(16)$ & $\mathrm{C} 15-\mathrm{C} 16$ & $1.3995(18)$ \\
\hline $\mathrm{C} 1-\mathrm{C} 6$ & $1.3812(18)$ & C15-H15 & $0.974(17)$ \\
\hline $\mathrm{C} 1-\mathrm{C} 2$ & $1.4098(17)$ & $\mathrm{C} 16-\mathrm{C} 17$ & $1.3828(19)$ \\
\hline $\mathrm{C} 2-\mathrm{C} 3$ & $1.3831(19)$ & $\mathrm{C} 16-\mathrm{C} 22$ & $1.5162(18)$ \\
\hline $\mathrm{C} 3-\mathrm{C} 4$ & $1.4025(18)$ & $\mathrm{C} 17-\mathrm{C} 18$ & $1.4015(19)$ \\
\hline $\mathrm{C} 3-\mathrm{H} 3$ & $0.984(16)$ & C17-H17 & $1.030(15)$ \\
\hline $\mathrm{C} 4-\mathrm{C} 5$ & $1.3833(19)$ & C18-H18 & $0.984(16)$ \\
\hline $\mathrm{C} 4-\mathrm{C} 10$ & $1.5099(18)$ & $\mathrm{C} 19-\mathrm{C} 20$ & $1.5148(16)$ \\
\hline $\mathrm{C} 5-\mathrm{C} 6$ & $1.4021(19)$ & C19-H19A & $1.012(15)$ \\
\hline $\mathrm{C} 5-\mathrm{H} 5$ & $1.005(16)$ & С19-Н19B & $0.992(16)$ \\
\hline C6- 66 & $0.972(16)$ & $\mathrm{C} 20-\mathrm{H} 20 \mathrm{~B}$ & $0.993(16)$ \\
\hline $\mathrm{C} 7-\mathrm{C} 8$ & $1.5154(16)$ & $\mathrm{C} 20-\mathrm{H} 20 \mathrm{~B}^{\mathrm{ii}}$ & $0.993(16)$ \\
\hline C7-H7A & $1.003(16)$ & $\mathrm{C} 21-\mathrm{H} 21 \mathrm{~A}$ & $1.013(17)$ \\
\hline C7-H7B & $1.007(15)$ & $\mathrm{C} 21-\mathrm{H} 21 \mathrm{~B}$ & $0.960(18)$ \\
\hline $\mathrm{C} 8-\mathrm{H} 8 \mathrm{~B}$ & $1.007(16)$ & $\mathrm{C} 21-\mathrm{H} 21 \mathrm{C}$ & $0.961(17)$ \\
\hline $\mathrm{C} 8-\mathrm{H} 8 \mathrm{~B}^{\mathrm{i}}$ & $1.007(16)$ & $\mathrm{C} 22-\mathrm{C} 23$ & $1.499(2)$ \\
\hline C9-H9A & 0.967 (17) & $\mathrm{C} 22-\mathrm{H} 22 \mathrm{~A}$ & $0.984(18)$ \\
\hline C9-H9B & $0.966(17)$ & $\mathrm{C} 22-\mathrm{H} 22 \mathrm{~B}$ & $1.010(17)$ \\
\hline $\mathrm{C} 9-\mathrm{H} 9 \mathrm{C}$ & $0.993(18)$ & $\mathrm{C} 23-\mathrm{C} 24$ & $1.315(2)$ \\
\hline $\mathrm{C} 10-\mathrm{C} 11$ & $1.507(2)$ & $\mathrm{C} 23-\mathrm{H} 23$ & $1.00(2)$ \\
\hline $\mathrm{C} 10-\mathrm{H} 10 \mathrm{~A}$ & 1.014 (17) & $\mathrm{C} 24-\mathrm{H} 24 \mathrm{~A}$ & $0.97(2)$ \\
\hline $\mathrm{C} 10-\mathrm{H} 10 \mathrm{~B}$ & $1.008(18)$ & $\mathrm{C} 24-\mathrm{H} 24 \mathrm{~B}$ & $0.97(2)$ \\
\hline $\mathrm{O} 1 \cdots \mathrm{O} 2$ & $2.5827(13)$ & $\mathrm{C} 14 \cdots \mathrm{H} 20 \mathrm{~B}^{\mathrm{iii}}$ & $2.918(15)$ \\
\hline $\mathrm{O} 2 \cdots \mathrm{O} 1$ & $2.5827(13)$ & $\mathrm{C} 15 \cdots \mathrm{H} 21 \mathrm{~A}$ & $2.768(17)$ \\
\hline $\mathrm{O} 3 \cdots \mathrm{O} 4$ & $2.5885(13)$ & $\mathrm{C} 15 \cdots \mathrm{H} 21 \mathrm{C}$ & $2.784(17)$ \\
\hline $\mathrm{O} 4 \cdots \mathrm{O} 3$ & $2.5885(13)$ & $\mathrm{C} 17 \cdots \mathrm{H} 22 \mathrm{~A}^{\mathrm{vi}}$ & 2.727 (19) \\
\hline $\mathrm{O} 1 \cdots \mathrm{H} 9 \mathrm{C}^{\mathrm{iii}}$ & $2.736(18)$ & $\mathrm{C} 18 \cdots \mathrm{H} 22 \mathrm{~A}^{\mathrm{vi}}$ & $2.764(18)$ \\
\hline $\mathrm{O} 1 \cdots \mathrm{H}^{\prime} \mathrm{B}^{\mathrm{i}}$ & $2.618(16)$ & 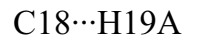 & $2.759(15)$ \\
\hline $\mathrm{O} 2 \cdots \mathrm{H} 12 \mathrm{~A}^{\mathrm{iv}}$ & $2.831(18)$ & C18ㄱH19B & $2.739(15)$ \\
\hline
\end{tabular}




\begin{tabular}{|c|c|c|c|}
\hline $\mathrm{O} 2 \cdots \mathrm{H} 22 \mathrm{~B}$ & $2.647(17)$ & $\mathrm{C} 19 \cdots \mathrm{H} 18$ & $2.514(16)$ \\
\hline $\mathrm{O} 2 \cdots \mathrm{H}_{8} \mathrm{~B}^{\mathrm{v}}$ & $2.676(15)$ & $\mathrm{C} 21 \cdots \mathrm{H} 15$ & $2.501(16)$ \\
\hline $\mathrm{O} 3 \cdots \mathrm{H} 21 \mathrm{~A}^{\mathrm{vi}}$ & $2.739(15)$ & $\mathrm{C} 23 \cdots \mathrm{H} 15$ & $2.862(16)$ \\
\hline O3 $\cdots H 19 A^{\mathrm{ii}}$ & $2.604(16)$ & H3 $\cdots$ H9C & $2.33(2)$ \\
\hline $\mathrm{O} 4 \cdots \mathrm{H} 24 \mathrm{~B}^{\mathrm{vii}}$ & $2.89(2)$ & H3 $\cdots$ H9A & $2.29(2)$ \\
\hline $\mathrm{O} 4 \cdots \mathrm{H} 20 \mathrm{~B}^{\mathrm{iii}}$ & $2.732(15)$ & $\mathrm{H} 5 \cdots \mathrm{H} 10 \mathrm{~A}$ & $2.37(2)$ \\
\hline $\mathrm{C} 2 \cdots \mathrm{C}^{\mathrm{vi}}$ & $3.533(2)$ & H6 $\cdots$ H7A & $2.24(2)$ \\
\hline $\mathrm{C} 3 \cdots \mathrm{C} 12$ & $3.282(2)$ & H6 $\cdots H 7 B$ & $2.37(2)$ \\
\hline $\mathrm{C} 6 \cdots \mathrm{C} 10^{\mathrm{iii}}$ & $3.582(2)$ & $\mathrm{H} 6 \cdots \mathrm{H} 18^{\text {viii }}$ & $2.50(2)$ \\
\hline $\mathrm{C} 14 \cdots \mathrm{C} 19^{\mathrm{iii}}$ & $3.555(2)$ & $\mathrm{H} 9 \mathrm{~A} \cdots \mathrm{H} 11^{\mathrm{ix}}$ & $2.40(2)$ \\
\hline $\mathrm{C} 18 \cdots \mathrm{C} 22^{\mathrm{vi}}$ & $3.564(2)$ & H9A $\cdots H 12 A^{\text {iv }}$ & $2.56(3)$ \\
\hline $\mathrm{C} 2 \cdots \mathrm{H} 8 \mathrm{~B}^{\mathrm{v}}$ & $2.914(16)$ & H9B $\cdots H 22 A^{v i}$ & $2.52(2)$ \\
\hline $\mathrm{C} 2 \cdots \mathrm{H} 7 \mathrm{~A}^{\mathrm{vi}}$ & $2.971(15)$ & H9B $\cdots \mathrm{H} 23$ & $2.41(2)$ \\
\hline $\mathrm{C} 3 \cdots \mathrm{H} 9 \mathrm{C}$ & $2.739(18)$ & $\mathrm{H} 9 \mathrm{C} \cdots \mathrm{H} 22 \mathrm{~B}^{\mathrm{vi}}$ & $2.54(2)$ \\
\hline C $3 \cdots \mathrm{H} 12 \mathrm{~B}$ & $2.783(19)$ & $\mathrm{H} 10 \mathrm{~A} \cdots \mathrm{H} 21 \mathrm{~A}^{\mathrm{x}}$ & $2.58(2)$ \\
\hline C3 $\cdots$ H9A & $2.806(17)$ & $\mathrm{H} 12 \mathrm{~B} \cdots \mathrm{H} 12 \mathrm{~B}^{\mathrm{iv}}$ & $2.55(3)$ \\
\hline $\mathrm{C} 4 \cdots \mathrm{H} 12 \mathrm{~B}$ & $2.728(18)$ & $\mathrm{H} 15 \cdots \mathrm{H} 21 \mathrm{~A}$ & $2.40(2)$ \\
\hline $\mathrm{C} 5 \cdots \mathrm{H} 10 \mathrm{~B}^{\mathrm{iii}}$ & $2.775(19)$ & $\mathrm{H} 15 \cdots \mathrm{H} 21 \mathrm{C}$ & $2.26(2)$ \\
\hline C6 $\cdots$ H7A & $2.719(15)$ & $\mathrm{H} 17 \cdots \mathrm{H} 22 \mathrm{~B}$ & $2.36(2)$ \\
\hline C $6 \cdots \mathrm{H} 7 \mathrm{~B}$ & $2.786(15)$ & 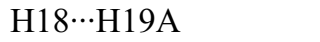 & $2.31(2)$ \\
\hline $\mathrm{C} 6 \cdots \mathrm{H} 10 \mathrm{~B}^{\mathrm{iii}}$ & $2.837(18)$ & 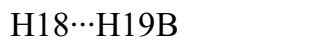 & $2.26(2)$ \\
\hline $\mathrm{C} 7 \cdots \mathrm{H} 6$ & $2.529(16)$ & $\mathrm{H} 21 \mathrm{C} \cdots \mathrm{H} 23^{\text {vii }}$ & $2.41(2)$ \\
\hline $\mathrm{C} 9 \cdots \mathrm{H} 3$ & $2.492(16)$ & $\mathrm{H} 22 \mathrm{~A} \cdots \mathrm{H} 24 \mathrm{~A}$ & $2.39(3)$ \\
\hline $\mathrm{C} 1-\mathrm{O} 1-\mathrm{C} 7$ & $116.96(10)$ & $\mathrm{C} 11-\mathrm{C} 12-\mathrm{H} 12 \mathrm{~B}$ & $123.1(11)$ \\
\hline $\mathrm{C} 2-\mathrm{O} 2-\mathrm{C} 9$ & $116.34(10)$ & $\mathrm{H} 12 \mathrm{~A}-\mathrm{C} 12-\mathrm{H} 12 \mathrm{~B}$ & $115.9(15)$ \\
\hline $\mathrm{C} 13-\mathrm{O} 3-\mathrm{C} 19$ & $116.88(10)$ & $\mathrm{O} 3-\mathrm{C} 13-\mathrm{C} 18$ & $125.61(11)$ \\
\hline $\mathrm{C} 14-\mathrm{O} 4-\mathrm{C} 21$ & $116.03(10)$ & $\mathrm{O} 3-\mathrm{C} 13-\mathrm{C} 14$ & $115.48(11)$ \\
\hline $\mathrm{O} 1-\mathrm{C} 1-\mathrm{C} 6$ & $125.38(11)$ & $\mathrm{C} 18-\mathrm{C} 13-\mathrm{C} 14$ & $118.90(11)$ \\
\hline $\mathrm{O} 1-\mathrm{C} 1-\mathrm{C} 2$ & $115.50(11)$ & $\mathrm{O} 4-\mathrm{C} 14-\mathrm{C} 15$ & $124.66(12)$ \\
\hline $\mathrm{C} 6-\mathrm{C} 1-\mathrm{C} 2$ & $119.12(11)$ & $\mathrm{O} 4-\mathrm{C} 14-\mathrm{C} 13$ & $115.42(11)$ \\
\hline $\mathrm{O} 2-\mathrm{C} 2-\mathrm{C} 3$ & $124.79(12)$ & $\mathrm{C} 15-\mathrm{C} 14-\mathrm{C} 13$ & $119.91(12)$ \\
\hline $\mathrm{O} 2-\mathrm{C} 2-\mathrm{C} 1$ & $115.21(11)$ & $\mathrm{C} 14-\mathrm{C} 15-\mathrm{C} 16$ & $121.15(12)$ \\
\hline $\mathrm{C} 3-\mathrm{C} 2-\mathrm{C} 1$ & $119.99(12)$ & $\mathrm{C} 14-\mathrm{C} 15-\mathrm{H} 15$ & $119.3(10)$ \\
\hline $\mathrm{C} 2-\mathrm{C} 3-\mathrm{C} 4$ & $121.06(12)$ & $\mathrm{C} 16-\mathrm{C} 15-\mathrm{H} 15$ & $119.6(10)$ \\
\hline $\mathrm{C} 2-\mathrm{C} 3-\mathrm{H} 3$ & $119.0(9)$ & $\mathrm{C} 17-\mathrm{C} 16-\mathrm{C} 15$ & $118.61(12)$ \\
\hline $\mathrm{C} 4-\mathrm{C} 3-\mathrm{H} 3$ & $119.9(9)$ & $\mathrm{C} 17-\mathrm{C} 16-\mathrm{C} 22$ & $121.67(12)$ \\
\hline $\mathrm{C} 5-\mathrm{C} 4-\mathrm{C} 3$ & $118.47(12)$ & $\mathrm{C} 15-\mathrm{C} 16-\mathrm{C} 22$ & $119.70(12)$ \\
\hline $\mathrm{C} 5-\mathrm{C} 4-\mathrm{C} 10$ & $121.09(12)$ & $\mathrm{C} 16-\mathrm{C} 17-\mathrm{C} 18$ & $120.79(12)$ \\
\hline $\mathrm{C} 3-\mathrm{C} 4-\mathrm{C} 10$ & $120.43(12)$ & $\mathrm{C} 16-\mathrm{C} 17-\mathrm{H} 17$ & $120.3(8)$ \\
\hline $\mathrm{C} 4-\mathrm{C} 5-\mathrm{C} 6$ & $120.97(12)$ & $\mathrm{C} 18-\mathrm{C} 17-\mathrm{H} 17$ & $118.9(9)$ \\
\hline $\mathrm{C} 4-\mathrm{C} 5-\mathrm{H} 5$ & $120.3(9)$ & $\mathrm{C} 13-\mathrm{C} 18-\mathrm{C} 17$ & $120.59(12)$ \\
\hline $\mathrm{C} 6-\mathrm{C} 5-\mathrm{H} 5$ & $118.7(9)$ & $\mathrm{C} 13-\mathrm{C} 18-\mathrm{H} 18$ & $119.4(9)$ \\
\hline $\mathrm{C} 1-\mathrm{C} 6-\mathrm{C} 5$ & $120.34(12)$ & $\mathrm{C} 17-\mathrm{C} 18-\mathrm{H} 18$ & $120.0(9)$ \\
\hline $\mathrm{C} 1-\mathrm{C} 6-\mathrm{H} 6$ & $120.2(9)$ & $\mathrm{O} 3-\mathrm{C} 19-\mathrm{C} 20$ & $107.42(9)$ \\
\hline $\mathrm{C} 5-\mathrm{C} 6-\mathrm{H} 6$ & $119.4(9)$ & $\mathrm{O} 3-\mathrm{C} 19-\mathrm{H} 19 \mathrm{~A}$ & $109.0(9)$ \\
\hline $\mathrm{O} 1-\mathrm{C} 7-\mathrm{C} 8$ & $107.63(9)$ & $\mathrm{C} 20-\mathrm{C} 19-\mathrm{H} 19 \mathrm{~A}$ & $112.1(8)$ \\
\hline $\mathrm{O} 1-\mathrm{C} 7-\mathrm{H} 7 \mathrm{~A}$ & $109.5(8)$ & $\mathrm{O} 3-\mathrm{C} 19-\mathrm{H} 19 \mathrm{~B}$ & $109.9(9)$ \\
\hline
\end{tabular}




\begin{tabular}{|c|c|}
\hline $\mathrm{C} 8-\mathrm{C} 7-\mathrm{H} 7 \mathrm{~A}$ & $110.4(9)$ \\
\hline $\mathrm{O} 1-\mathrm{C} 7-\mathrm{H} 7 \mathrm{~B}$ & $109.4(9)$ \\
\hline $\mathrm{C} 8-\mathrm{C} 7-\mathrm{H} 7 \mathrm{~B}$ & $111.6(9)$ \\
\hline $\mathrm{H} 7 \mathrm{~A}-\mathrm{C} 7-\mathrm{H} 7 \mathrm{~B}$ & $108.2(12)$ \\
\hline $\mathrm{C} 7-\mathrm{C} 8-\mathrm{C}^{\mathrm{i}}$ & $114.11(15)$ \\
\hline $\mathrm{C} 7-\mathrm{C} 8-\mathrm{H} 8 \mathrm{~B}$ & $106.1(9)$ \\
\hline $\mathrm{C} 7^{\mathrm{i}}-\mathrm{C} 8-\mathrm{H} 8 \mathrm{~B}$ & $110.0(9)$ \\
\hline $\mathrm{C} 7-\mathrm{C} 8-\mathrm{H} 8 \mathrm{~B}^{\mathrm{i}}$ & $110.0(9)$ \\
\hline $\mathrm{C}^{\mathrm{i}}-\mathrm{C} 8-\mathrm{H} 8 \mathrm{~B}^{\mathrm{i}}$ & $106.1(9)$ \\
\hline $\mathrm{H} 8 \mathrm{~B}-\mathrm{C} 8-\mathrm{H} 8 \mathrm{~B}^{\mathrm{i}}$ & $110.5(18)$ \\
\hline $\mathrm{O} 2-\mathrm{C} 9-\mathrm{H} 9 \mathrm{~A}$ & $111.2(10)$ \\
\hline $\mathrm{O} 2-\mathrm{C} 9-\mathrm{H} 9 \mathrm{~B}$ & $104.5(9)$ \\
\hline $\mathrm{H} 9 \mathrm{~A}-\mathrm{C} 9-\mathrm{H} 9 \mathrm{~B}$ & $109.1(13)$ \\
\hline $\mathrm{O} 2-\mathrm{C} 9-\mathrm{H} 9 \mathrm{C}$ & $110.3(10)$ \\
\hline $\mathrm{H} 9 \mathrm{~A}-\mathrm{C} 9-\mathrm{H} 9 \mathrm{C}$ & $111.2(14)$ \\
\hline $\mathrm{H} 9 \mathrm{~B}-\mathrm{C} 9-\mathrm{H} 9 \mathrm{C}$ & $110.5(13)$ \\
\hline $\mathrm{C} 11-\mathrm{C} 10-\mathrm{C} 4$ & $116.06(12)$ \\
\hline $\mathrm{C} 11-\mathrm{C} 10-\mathrm{H} 10 \mathrm{~A}$ & $108.5(9)$ \\
\hline $\mathrm{C} 4-\mathrm{C} 10-\mathrm{H} 10 \mathrm{~A}$ & $109.5(10)$ \\
\hline $\mathrm{C} 11-\mathrm{C} 10-\mathrm{H} 10 \mathrm{~B}$ & $106.7(10)$ \\
\hline $\mathrm{C} 4-\mathrm{C} 10-\mathrm{H} 10 \mathrm{~B}$ & $108.3(10)$ \\
\hline $\mathrm{H} 10 \mathrm{~A}-\mathrm{C} 10-\mathrm{H} 10 \mathrm{~B}$ & $107.5(14)$ \\
\hline $\mathrm{C} 12-\mathrm{C} 11-\mathrm{C} 10$ & $127.94(14)$ \\
\hline $\mathrm{C} 12-\mathrm{C} 11-\mathrm{H} 11$ & $118.0(11)$ \\
\hline $\mathrm{C} 10-\mathrm{C} 11-\mathrm{H} 11$ & $114.0(11)$ \\
\hline $\mathrm{C} 11-\mathrm{C} 12-\mathrm{H} 12 \mathrm{~A}$ & $121.0(11)$ \\
\hline $\mathrm{C} 7-\mathrm{O} 1-\mathrm{C} 1-\mathrm{C} 6$ & $-3.97(17)$ \\
\hline $\mathrm{C} 7-\mathrm{O} 1-\mathrm{C} 1-\mathrm{C} 2$ & $175.08(10)$ \\
\hline $\mathrm{C} 9-\mathrm{O} 2-\mathrm{C} 2-\mathrm{C} 3$ & $11.70(18)$ \\
\hline $\mathrm{C} 9-\mathrm{O} 2-\mathrm{C} 2-\mathrm{C} 1$ & $-167.55(11)$ \\
\hline $\mathrm{O} 1-\mathrm{C} 1-\mathrm{C} 2-\mathrm{O} 2$ & $-1.88(16)$ \\
\hline $\mathrm{C} 6-\mathrm{C} 1-\mathrm{C} 2-\mathrm{O} 2$ & $177.24(11)$ \\
\hline $\mathrm{O} 1-\mathrm{C} 1-\mathrm{C} 2-\mathrm{C} 3$ & $178.83(11)$ \\
\hline $\mathrm{C} 6-\mathrm{C} 1-\mathrm{C} 2-\mathrm{C} 3$ & $-2.05(18)$ \\
\hline $\mathrm{O} 2-\mathrm{C} 2-\mathrm{C} 3-\mathrm{C} 4$ & $-178.74(12)$ \\
\hline $\mathrm{C} 1-\mathrm{C} 2-\mathrm{C} 3-\mathrm{C} 4$ & $0.48(19)$ \\
\hline $\mathrm{C} 2-\mathrm{C} 3-\mathrm{C} 4-\mathrm{C} 5$ & $1.21(19)$ \\
\hline $\mathrm{C} 2-\mathrm{C} 3-\mathrm{C} 4-\mathrm{C} 10$ & $-179.70(12)$ \\
\hline $\mathrm{C} 3-\mathrm{C} 4-\mathrm{C} 5-\mathrm{C} 6$ & $-1.35(19)$ \\
\hline $\mathrm{C} 10-\mathrm{C} 4-\mathrm{C} 5-\mathrm{C} 6$ & $179.57(12)$ \\
\hline $\mathrm{O} 1-\mathrm{C} 1-\mathrm{C} 6-\mathrm{C} 5$ & $-179.06(11)$ \\
\hline $\mathrm{C} 2-\mathrm{C} 1-\mathrm{C} 6-\mathrm{C} 5$ & $1.92(19)$ \\
\hline $\mathrm{C} 4-\mathrm{C} 5-\mathrm{C} 6-\mathrm{C} 1$ & $-0.22(19)$ \\
\hline $\mathrm{C} 1-\mathrm{O} 1-\mathrm{C} 7-\mathrm{C} 8$ & $178.57(10)$ \\
\hline $\mathrm{O} 1-\mathrm{C} 7-\mathrm{C} 8-\mathrm{C}^{\mathrm{i}}$ & $56.52(7)$ \\
\hline $\mathrm{C} 5-\mathrm{C} 4-\mathrm{C} 10-\mathrm{C} 11$ & $-113.66(15)$ \\
\hline
\end{tabular}

\begin{tabular}{|c|c|}
\hline $\mathrm{C} 20-\mathrm{C} 19-\mathrm{H} 19 \mathrm{~B}$ & $110.6(9)$ \\
\hline $\mathrm{H} 19 \mathrm{~A}-\mathrm{C} 19-\mathrm{H} 19 \mathrm{~B}$ & $107.9(12)$ \\
\hline $\mathrm{C} 19-\mathrm{C} 20-\mathrm{C} 19^{\mathrm{ii}}$ & $113.61(15)$ \\
\hline $\mathrm{C} 19-\mathrm{C} 20-\mathrm{H} 20 \mathrm{~B}$ & $110.4(9)$ \\
\hline $\mathrm{C} 19^{\mathrm{ii}}-\mathrm{C} 20-\mathrm{H} 20 \mathrm{~B}$ & $107.3(9)$ \\
\hline $\mathrm{C} 19-\mathrm{C} 20-\mathrm{H} 20 \mathrm{~B}^{\mathrm{ii}}$ & $107.3(9)$ \\
\hline $\mathrm{C} 19^{\mathrm{ii}}-\mathrm{C} 20-\mathrm{H} 20 \mathrm{~B}^{\mathrm{ii}}$ & $110.4(9)$ \\
\hline $\mathrm{H} 20 \mathrm{~B}-\mathrm{C} 20-\mathrm{H} 20 \mathrm{~B}^{\mathrm{ii}}$ & $107.6(18)$ \\
\hline $\mathrm{O} 4-\mathrm{C} 21-\mathrm{H} 21 \mathrm{~A}$ & $110.7(9)$ \\
\hline $\mathrm{O} 4-\mathrm{C} 21-\mathrm{H} 21 \mathrm{~B}$ & $105.3(10)$ \\
\hline $\mathrm{H} 21 \mathrm{~A}-\mathrm{C} 21-\mathrm{H} 21 \mathrm{~B}$ & $109.0(14)$ \\
\hline $\mathrm{O} 4-\mathrm{C} 21-\mathrm{H} 21 \mathrm{C}$ & $111.0(10)$ \\
\hline $\mathrm{H} 21 \mathrm{~A}-\mathrm{C} 21-\mathrm{H} 21 \mathrm{C}$ & $111.5(14)$ \\
\hline $\mathrm{H} 21 \mathrm{~B}-\mathrm{C} 21-\mathrm{H} 21 \mathrm{C}$ & $109.1(13)$ \\
\hline $\mathrm{C} 23-\mathrm{C} 22-\mathrm{C} 16$ & $113.46(11)$ \\
\hline $\mathrm{C} 23-\mathrm{C} 22-\mathrm{H} 22 \mathrm{~A}$ & $107.1(10)$ \\
\hline $\mathrm{C} 16-\mathrm{C} 22-\mathrm{H} 22 \mathrm{~A}$ & $109.6(10)$ \\
\hline $\mathrm{C} 23-\mathrm{C} 22-\mathrm{H} 22 \mathrm{~B}$ & $109.9(9)$ \\
\hline $\mathrm{C} 16-\mathrm{C} 22-\mathrm{H} 22 \mathrm{~B}$ & $108.1(10)$ \\
\hline $\mathrm{H} 22 \mathrm{~A}-\mathrm{C} 22-\mathrm{H} 22 \mathrm{~B}$ & $108.7(14)$ \\
\hline $\mathrm{C} 24-\mathrm{C} 23-\mathrm{C} 22$ & $125.43(15)$ \\
\hline $\mathrm{C} 24-\mathrm{C} 23-\mathrm{H} 23$ & $119.7(11)$ \\
\hline $\mathrm{C} 22-\mathrm{C} 23-\mathrm{H} 23$ & $114.8(11)$ \\
\hline $\mathrm{C} 23-\mathrm{C} 24-\mathrm{H} 24 \mathrm{~A}$ & $120.3(11)$ \\
\hline $\mathrm{C} 23-\mathrm{C} 24-\mathrm{H} 24 \mathrm{~B}$ & $122.2(12)$ \\
\hline $\mathrm{H} 24 \mathrm{~A}-\mathrm{C} 24-\mathrm{H} 24 \mathrm{~B}$ & $117.5(17)$ \\
\hline $\mathrm{C} 19-\mathrm{O} 3-\mathrm{C} 13-\mathrm{C} 18$ & $2.61(18)$ \\
\hline $\mathrm{C} 19-\mathrm{O} 3-\mathrm{C} 13-\mathrm{C} 14$ & $-176.67(10$ \\
\hline $\mathrm{C} 21-\mathrm{O} 4-\mathrm{C} 14-\mathrm{C} 15$ & $-14.30(18)$ \\
\hline $\mathrm{C} 21-\mathrm{O} 4-\mathrm{C} 14-\mathrm{C} 13$ & $164.57(11)$ \\
\hline $\mathrm{O} 3-\mathrm{C} 13-\mathrm{C} 14-\mathrm{O} 4$ & $2.86(16)$ \\
\hline $\mathrm{C} 18-\mathrm{C} 13-\mathrm{C} 14-\mathrm{O} 4$ & $-176.47(11$ \\
\hline $\mathrm{O} 3-\mathrm{C} 13-\mathrm{C} 14-\mathrm{C} 15$ & $-178.22(11$ \\
\hline $\mathrm{C} 18-\mathrm{C} 13-\mathrm{C} 14-\mathrm{C} 15$ & $2.45(18)$ \\
\hline $\mathrm{O} 4-\mathrm{C} 14-\mathrm{C} 15-\mathrm{C} 16$ & $177.52(12)$ \\
\hline $\mathrm{C} 13-\mathrm{C} 14-\mathrm{C} 15-\mathrm{C} 16$ & $-1.29(19)$ \\
\hline $\mathrm{C} 14-\mathrm{C} 15-\mathrm{C} 16-\mathrm{C} 17$ & $-0.69(19)$ \\
\hline $\mathrm{C} 14-\mathrm{C} 15-\mathrm{C} 16-\mathrm{C} 22$ & $-178.93(12$ \\
\hline $\mathrm{C} 15-\mathrm{C} 16-\mathrm{C} 17-\mathrm{C} 18$ & $1.52(19)$ \\
\hline $\mathrm{C} 22-\mathrm{C} 16-\mathrm{C} 17-\mathrm{C} 18$ & $179.71(12)$ \\
\hline $\mathrm{O} 3-\mathrm{C} 13-\mathrm{C} 18-\mathrm{C} 17$ & $179.10(12)$ \\
\hline $\mathrm{C} 14-\mathrm{C} 13-\mathrm{C} 18-\mathrm{C} 17$ & $-1.65(19)$ \\
\hline $\mathrm{C} 16-\mathrm{C} 17-\mathrm{C} 18-\mathrm{C} 13$ & $-0.34(19)$ \\
\hline $\mathrm{C} 13-\mathrm{O} 3-\mathrm{C} 19-\mathrm{C} 20$ & $-179.34(10$ \\
\hline $\mathrm{O} 3-\mathrm{C} 19-\mathrm{C} 20-\mathrm{C} 19^{\mathrm{ii}}$ & $-56.61(7)$ \\
\hline $\mathrm{C} 17-\mathrm{C} 16-\mathrm{C} 22-\mathrm{C} 23$ & $126.83(14)$ \\
\hline
\end{tabular}




\section{supporting information}

$\begin{array}{llll}\mathrm{C} 3-\mathrm{C} 4-\mathrm{C} 10-\mathrm{C} 11 & 67.28(17) & \mathrm{C} 15-\mathrm{C} 16-\mathrm{C} 22-\mathrm{C} 23 & -55.00(17) \\ \mathrm{C} 4-\mathrm{C} 10-\mathrm{C} 11-\mathrm{C} 12 & -1.4(2) & \mathrm{C} 16-\mathrm{C} 22-\mathrm{C} 23-\mathrm{C} 24 & 111.83(17)\end{array}$

Symmetry codes: (i) $-x+3 / 2, y,-z+1 / 2$; (ii) $-x+1 / 2, y,-z+1 / 2$; (iii) $x, y-1, z$; (iv) $-x+2,-y,-z+1$; (v) $-x+3 / 2, y+1,-z+1 / 2$; (vi) $x, y+1, z$; (vii) $-x+1,-y$, $-z+1$; (viii) $-x+3 / 2, y-1,-z+1 / 2$; (ix) $-x+2,-y+1,-z+1$; (x) $x+1, y+1, z$.

Hydrogen-bond geometry $\left(\AA,{ }^{\circ}\right)$

$C g 1$ and $C g 2$ are the centroids of benzene rings $A(\mathrm{C} 1-\mathrm{C} 6)$ and $B(\mathrm{C} 13-\mathrm{C} 18)$, respectively.

\begin{tabular}{lllll}
\hline$D-\mathrm{H} \cdots A$ & $D-\mathrm{H}$ & $\mathrm{H} \cdots A$ & $D \cdots A$ & $D-\mathrm{H} \cdots A$ \\
\hline $\mathrm{C} 7-\mathrm{H} 7 A \cdots C g 1^{\mathrm{iii}}$ & $1.003(16)$ & $2.759(15)$ & $3.6170(15)$ & $144.1(12)$ \\
$\mathrm{C} 19-\mathrm{H} 19 B \cdots C g 2^{\mathrm{vi}}$ & $0.992(16)$ & $2.739(15)$ & $3.5816(15)$ & $143.0(11)$ \\
\hline
\end{tabular}

Symmetry codes: (iii) $x, y-1, z$; (vi) $x, y+1, z$. 